\section{Clinical ocular pharmacology}

Jimmy D Bartlett and Siret D Jaanus

Butterworth-Heinemann, 4th

edition, 2001, £70 ISBN 0750670398

Eye (2002) 16, 681.

doi:10.1038/sj.eye.6700052

Bigger and brighter than the third edition this is a most welcome update to what is justifiably considered the gold standard in basic pharmacology of the eye.

This substantial book consisting of 38 chapters weighs 3 kilograms and has 48 contributing authors. Chapters are grouped into four parts, the fundamentals of ocular pharmacology; the pharmacology of ocular drugs; ocular drugs used in clinical practice and ocular toxicology.

Inclusion of the non steroidal anti-inflammatory drugs (NSAIDs) with selectivity for the cox-2 iso enzymes is most welcome in the chapter on analgesics as is some structured guidance in the first chapter where use of topical drugs in pregnancy looks at current FDA classification.

In the second part of this new edition a chapter on the pharmacological management of refractive surgery replaces a topic on contact lens care reflecting current trends within the speciality.

Glaucoma drugs are updated to include brinzolamide and a summary of the clinical advantages of topical carbonic anhydrase inhibitors (CAIs) whilst the prostaglandin section discusses the use of latanoprost in greater depth and includes the newer agents unoprostone and travoprost. The section on glaucoma management includes updated prescribing advice which excludes pilocarpine and focuses on beta blockers, brimonidine, carbonic anhydrase inhibitors or a combination of two or more of these. The classification of anti-infective agents now includes mechanisms of action and the chapter on the retina gives a brief explanation of photodynamic therapy with appropriate references.

There are significant useful additions to the chapter on ocular lubricants, in particular a chart classification of dry eye conditions plus a section on tear stimulation as a future therapeutic option. The chapter on allergic eye disease has been significantly improved by providing a clear introduction to immunology with the different types of allergic hypersensitivity reactions and has useful tables for ease of reference to both disease states and treatment options.

Section four on toxicology covers drug interactions and the data in this new edition are more succinct detailing only those of clinical significance. Ocular effects of systemic drugs include rifabutin associated uveitis and ocular changes with sildenafil and vigabatrin. In this edition there is an emphasis on the need to report any suspected adverse events although the mechanism for reporting is only relevant to the USA.

The final chapter on the management of systemic emergencies has been expanded to include drug-related reactions and offers defensive strategies to prevent or minimise adverse drug events. Each chapter is well supported by references with an emphasis on sources from peerreviewed literature.

The attractive style with bolder print has meant some compromises where text shown in tables has been abbreviated and where for this latest edition comparative figures have not been reproduced on adjacent pages.
Although the use of chemical structures may not be considered clinically relevant I would like to have seen a few more retained. Structures of antivirals in the previous edition, for example, usefully highlight structural similarities to target DNA nucleosides.

Some data, such as the chart of commonly used abbreviations is only relevant to American practice and indeed use of these in the UK could lead to dispensing errors. Another minor criticism is the occasional inconsistency with trade and generic drug nomenclature.

This invaluable book, with a wealth of reference material is relevant to all ophthalmic professionals, and well worth the price.

$$
\begin{array}{r}
\text { V Haylor } \\
\text { Ophthalmic Pharmacist } \\
\text { Royal Hallamshire Hospital } \\
\text { Sheffield, UK }
\end{array}
$$

\section{Binocular vision and orthoptics- investigation and management \\ B Evans and S Doshi \\ Butterworth-Heinemann, Oxford, 2001 \\ $£ 39.99$, ISBN 0750647132}

Eye (2002) 16, 681-682.

doi:10.1038/sj.eye. 6700185

This publication is a compilation of 16 articles that were published by various authors in the journal Optician between 1998 and 2000. The articles have been updated to form 16 chapters. There are 11 contributors, mainly optometrists but also an ophthalmologist and orthoptist.

Binocular Vision and Orthoptics is specifically aimed at eye-care 